Case Report

Avinash Chandra, MD

Annapurna Neurological Institute and Allied Sciences

Kathmandu, Nepal

Reema Rajbhandari, MD

Annapurna Neurological Institute and Allied Sciences \& Institute of Medicine, Tribhuvan University

Kathmandu, Nepal

Samir Acharya, MS

Annapurna Neurological Institute and Allied Sciences

Kathmandu, Nepal

Priya Gurung, MBBS

Annapurna Neurological Institute and Allied Sciences

Kathmandu, Nepal

Basant Pant, MD, PhD

Annapurna Neurological Institute and Allied Sciences

Kathmandu, Nepal

Address for correspondence:

Avinash Chandra, MD

Annapurna Neurological Institute and Allied Sciences

Kathmandu, Nepal

Email: chandraavi@gmail.com

Received, 16 September, 2016

Accepted, 29 September, 2016

A cute transverse myelitis is a focal inflammatory disorder of the spinal cord. ${ }^{3}$ One of the main etiologic factors include, multiple sclerosis, postinfectious and post-traumatic events ${ }^{7}$ although autoimmune phenomenon is the most common cause. ${ }^{7}$ Transverse Myelitis (TM) due to etiology other than Multiple Sclerosis has shown spinal cord involvement of two or more segments. ${ }^{5}$ Annually millions of active immunization with vaccines is carried out globally with few transverse myelitis caused because of the adverse reactions due to these vaccinations. ${ }^{2,9}$ In order to improve accuracy of reporting, research and diagnosis, the Transverse Myelitis Consortium Working Group (TMCWG) have produced criteria for the diagnosis of ATM. ${ }^{11}$

Here we report a case of TM in adult, diagnosed as TM consistent with the TMCWG criteria and probably this is the first ever been reported case from Nepal.
Nepal Journal of Neuroscience 13:89-91, 2016

\section{Vaccine Induced Acute Transverse Myelitis: A Case Report}

Acute transverse myelitis is a focal inflammatory disorder of the spinal cord. One of the main etiologic factors include, multiple sclerosis, post-infectious and post-traumatic events although autoimmune phenomenon is the most common cause. ${ }^{2}$ Transverse Myelitis (TM) due to etiology other than Multiple Sclerosis has shown spinal cord involvement of two or more segments. Annually millions of active immunization with vaccines is carried out globally with few transverse myelitis caused because of the adverse reactions due to these vaccinations. In order to improve accuracy of reporting, research and diagnosis, the Transverse Myelitis Consortium Working Group (TMCWG) have produced criteria for the diagnosis of ATM. Here we report a case of TM in adult, diagnosed as TM consistent with the TMCWG criteria and probably this is the first ever been reported case from Nepal.

Key Words: acute transverse myelitis, autoimmune, vaccine

\section{Case Report}

An otherwise healthy 38-year-old male presented to the Neurology clinic with the complaint of progressive weakness of both of his lower limbs that began 2 days earlier. Before the onset of weakness, he felt mild pain associated with the tingling sensation from the waist down. Several hours later after that he felt difficulty in passing urine and burning sensation while passing it eventually urinary urgency. He visited the local hospital and was prescribed metronidazole and pain killer with the diagnosis of Urinary Tract Infection (UTI). Symptoms, however, did not get relieved despite medications. On the next morning, he noticed that he was able to raise his legs only with great difficulty. Weakness progressed ultimately resulting to an inability to stand. He also noted that he had numbness below his nipple line and neck flexon triggered 

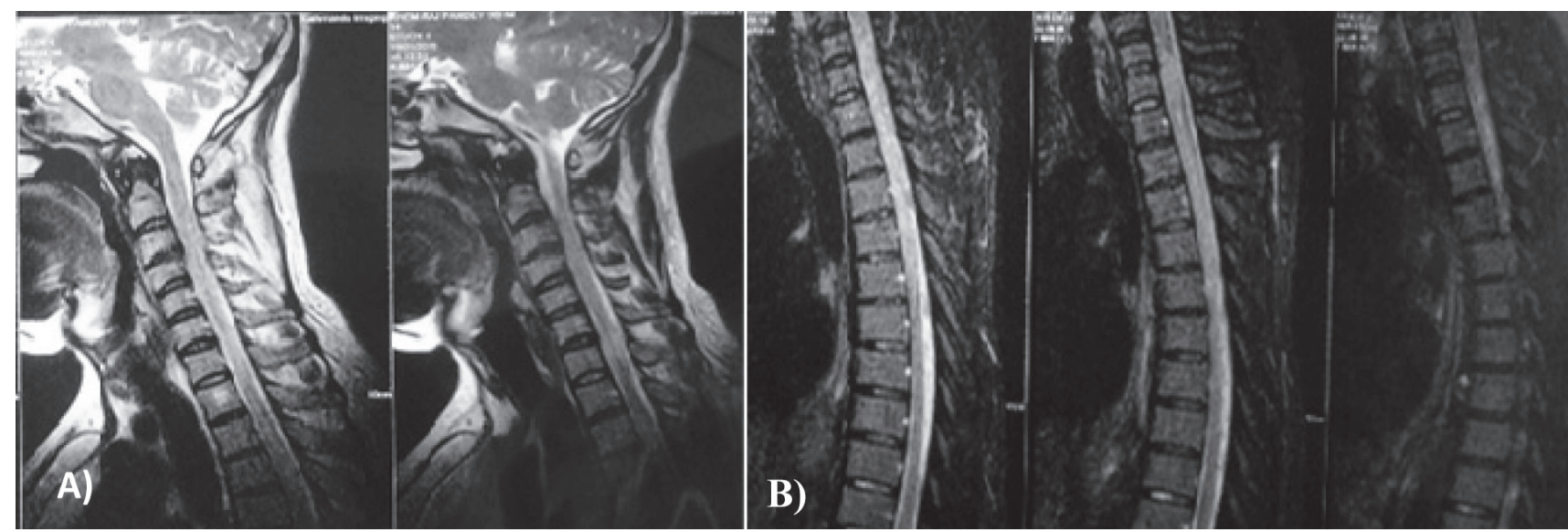

Figure 1: Sagital images of the spine demonstrating diffuse high signal in T2WI at the level of lower cervical and upper dorsal spine

an electrical like sensation going till coccyx. Ten days prior to the onset of these symptoms, he had received vaccination for MMR and Influenza as a part of vaccination program before immigration to the USA. Examination revealed he was apyrexic, with normal fundi, cranial nerves and upper limbs. Examination of lower limbs demonstrated slightly reduced tone, a pyramidal pattern of weakness, and moderately brisk reflexes with ankle clonus and extensor plantars of bilateral lower limbs. Sensory examination showed diminished sensation below T5/6 without the clear cut level. Abdominal reflexes were absent. Initial investigations revealed mild neutrophilic leukocytes and slightly raised ESR. Other routine investigations were within normal limits. An urgent MRI scan of the spinal cord was performed. No epidural collection or herniated disc or discitis was noted. However there was abnormal signal noted within the cord revealing the short segment patchy area of high intensity in cervical spinal cord at C7 to D5 levels (Figure 1). Muscle Research Council (MRC) scale showed muscle power bilateral upper limb as normal and bilateral lower limb as $1 / 5$. Cerebrospinal fluid had a protein concentration of $20 \mathrm{mg} / \mathrm{dl}$, a glucose concentration of $3.5 \mathrm{mmol} / 1$, and a white blood cell count of $1 / \mathrm{cumm}$ with $100 \%$ lymphocytes. Bacterial and viral cultures as well as oligoclonal bands results were negative. All other lab investigations revealed no abnormality.

Steroid with high dose $(\sim 15 \mathrm{mg} / \mathrm{kg}$ body weight) followed by rapid tapering ( $\sim 2$ weeks) and then pulse therapy for another 2 weeks was used as a main line treatment besides giving other supplements like calcium. Therapy also included physiotherapy along with the acupuncture therapy.

\section{Discussion}

Ever since the age of vaccine has started, the morbidity and mortality caused by many infectious diseases has been successfully declining over time. The function of vaccines is to initiate and produce antigen-specific $\operatorname{IgG}$ antibodies and stimulate body's immune system without actually causing the disease. However despite the usefulness of vaccines, attention has now shifted to the complications that arise after vaccination. Several complications related to neurological conditions like Guillian Barr Syndrome, Transverse Myelitis, Neuromyelitis Optica, Acute Disseminated Encephalopathy are being reported with several vaccines though not proven. The probable diagnoses are based on biological and laboratory evidences or in some cases epidemiologic evidences. ${ }^{16}$ TMCWG criteria states that in order to diagnose as TM, there needs to be exclusion of idiopathic TM, Bilateral signs and/or symptoms (though not necessarily symmetric), exclusion of extra-axial compressive etiology by neuroimaging. ${ }^{11}$ Our case fits well with these criteria since our case has dysautonomic function and MRI evidence of diffusely high signal in D5 level and onward. There are some hypotheses regarding the possibility of TM following vaccination as this is not a usual adverse event ${ }^{8,6,4}$ and the causal relationship of vaccination with TM has not yet been well established. ${ }^{15}$ Only few (around 5) cases of TM following Tetanus toxoid (Td) and diphtheriatetanus-pertussis (DTP) as a single vaccine or in various combinations have been reported so far. ${ }^{17,12}$ Out of those cases, only one is TM following Td vaccination. ${ }^{1,6,12,13,17}$ The hypotheses for vaccine induced neuroinflammatory disorder emphasize on the concept of autoimmunity where there is cross reaction of antibodies and $\mathrm{T}$ cells to CNS and PNS neural epitopes. ${ }^{14}$ There is another, "Molecular Mimicry" hypothesis which basically states that immunization may result in autoimmune disease because the proteins on microbial pathogens are similar to the human proteins and thus induce immune response that damage the human cells ${ }^{15}$ with some conflicting reports on animal studies. ${ }^{14}$ 


\section{Conclusion}

Transverse myelitis is an inflammatory disorders that are characterized by acute or sub acute motor, sensory, and autonomic (bladder, bowel, and sexual) spinal cord dysfunction. Demyelinating diseases (of neuroinflammatory origins) might also be caused due to the cross reaction from vaccines, although very less likely. However, to establish the causal relationship, large based case studies, with studies on animal model and molecular level are needed.

\section{References}

1. Abdul-Ghaffar NU, Achar KN. Brown-Sequard syndrome following diphtheria and tetanus vaccines.

Trop Doct 24:74-75, 1994

2. Cizman M, Pokorn M, Osredkar D. Re: transverse myelitis after measles and rubella vaccination. J Paediatr Child Health 41:460, 2005

3. de Seze J, Stojkovic T, Breteau G, et al. Acute myelopathies: Clinical, laboratory and outcome profiles in 79 cases. Brain : a journal of neurology 124:1509-1521, 2001

4. Foley KM, Beresford HR. Acute poliomyelitis beginning as transverse myelopathy. Archives of neurology 30:182-183, 1974

5. Harzheim M, Schlegel U, Urbach H, Klockgether T, Schmidt S. Discriminatory features of acute transverse myelitis: a retrospective analysis of 45 patients. J Neurol Sci 217:217-223, 2004

6. Kelly H. Evidence for a causal association between oral polio vaccine and transverse myelitis: A case history and review of the Literature. J Paediatr Child Health 42:155-159, 2006

7. Krishnan C, Kaplin AI, Deshpande DM, Pardo

\section{Transverse Myelitis}

CA, Kerr DA. Transverse Myelitis: pathogenesis, diagnosis and treatment. Front Biosci 9:1483-1499, 2004

8. Lance JW, White SW, Dodgson MC. Sensory Loss in Poliomyelitis. Australas Ann Med 12:221-229, 1963

9. Lim S, Park SM, Choi HS, et al. Transverse myelitis after measles and rubella vaccination. J Paediatr Child Health 40:583-584, 2004

10. Read SJ, Schapel GJ, Pender MP. Acute transverse myelitis after tetanus toxoid vaccination. Lancet 339:1111-1112, 1992

11. Riel-Romero RM. Acute transverse myelitis in a 7-month-old boy after diphtheria-tetanus-pertussis immunization. Spinal Cord 44:688-691, 2006

12. Shoenfeld Y, Aron-Maor A. Vaccination and autoimmunity-'vaccinosis': a dangerous liaison? J Autoimmun 14:1-10, 2000

13. Salemi S, D'Amelio R. Could autoimmunity be induced by vaccination? Int Rev Immunol 29:247269, 2010

14. Transverse Myelitis Consortium Working Group. Proposed diagnostic criteria and nosology of acute transverse myelitis. Neurology 59:499-505, 2002

15. Update: vaccine side effects, adverse reactions, contraindications, and precautions. Recommendations of the Advisory Committee on Immunization Practices (ACIP). MMWR Recomm Rep 45:1-35, 1996

16. von Geldern G, McPharlin T, Becker K. Immune mediated diseases and immune modulation in the neurocritical care unit. Neurotherapeutics 9:99123, 2012

17. Whittle E, Robertson NR. Transverse myelitis after diphtheria, tetanus, and polio immunisation. Br Med J 1:1450, 1977 